\section{Criteria for CAPS, is it all in the name?}

This paper ${ }^{1}$ describes an original work conducted by an International team of 16 recognised clinical experts in the field of autoinflammatory diseases. The aim of this consortium was to develop diagnostic criteria for cryopyrin-associated periodic syndrome (CAPS). They resulted in a model that indisputably is relevant to describe these rare and heterogeneous diseases among other autoinflammatory diseases. Their proposed CAPS diagnosis criteria are primarily clinical.

We would like to comment on the pathophysiological mechanism underlying 'CAPS'. The NLRP3 gene encodes cryopyrin, the historical name of the NLRP3 protein, a key component of the NLRP3 inflammasome. ${ }^{2}$ As clearly stated by the authors, 'cryopyrin-associated periodic syndrome' includes, by definition, a group of diseases associated with NLRP3 mutations. Deriving from this concept, a 'non-CAPS autoinflammatory disease' would correspond to a disease caused by mutations/polymorphisms in one or several other gene(s). The consortium nevertheless concluded that 'most importantly', the model 'did not mandate evidence of a disease-causing NLRP3 mutation'. Indeed, somatic mosaicism can be missed by conventional sequencing approaches. ${ }^{3}$ However, the proposed criteria cannot stricto sensu be restricted to CAPS as it is likely to encompass syndromes caused by mutations in autoinflammatory genes other than NLRP3. For example, familial cold autoinflammatory syndromes (FCAS) can result from a number of recently discovered genes. FCAS1 (online inheritance in men (OMIM) no. 120100 ) is the classical FCAS caused by mutations in NLRP3; FCAS2 (OMIM no. 611762, also known as NLRP12-associated periodic syndrome (NAPS12), NLRP12-associated periodic syndrome), FCAS3 (OMIM no. 614468, also known as PLCG2associated antibody deficiency and immune dysregulation (PLAID), Phospholipase C Gamma 2 (PLCG2)-associated antibody deficiency and immune dysregulation) and FCAS4 (OMIM no. 616115, also known as AIFEC, autoinflammation with infantile enterocolitis or recurrent macrophage activation syndrome) are due to the mutations in NLRP12, PLCG2 and NLRC4, respectively. The proposed criteria (raised inflammatory markers, urticaria-like rash and arthralgia) would perform well with these four FCAS, but only FCAS1 is a CAPS. The authors fairly admitted that 'the number of CAPS cases and controls was limited and not all possible differential diagnoses of CAPS may have been included, potentially leading to an overestimation of the specificity of the proposed model'.

The issue of the disease name, reflecting either the main symptoms or the molecular mechanisms of the condition, has been raised many times in the autoinflammatory diseases community, and propositions for refined taxonomy will shortly emerge. The debate is not just semantic as differential therapeutic approaches can be taken according to the molecular defect in cause in the condition presented by the patient.

\section{Isabelle Touitou, Guillaume Sarrabay}

Reference Centre for Autoinflammatory Diseases, Hôpital A de Villeneuve, CHRU Montpellier, Montpellier, France

Correspondence to Professor Isabelle Touitou, Reference Centre for Autoinflammatory Diseases, Hôpital A de Villeneuve, CHRU Montpellier, 34295 Montpellier Cedex 5, France; Isabelle.touitou@inserm.fr

Competing interests None.

Provenance and peer review Not commissioned; internally peer reviewed.

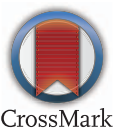

To cite Touitou I, Sarrabay G. Ann Rheum Dis 2017;76:e9.

Received 14 October 2016

Accepted 18 October 2016

Published Online First 16 November 2016

Ann Rheum Dis 2017;76:e9. doi:10.1136/annrheumdis-2016-210681

\section{REFERENCES}

1 Kuemmerle-Deschner JB, Ozen S, Tyrrell PN, et al. Diagnostic criteria for cryopyrin-associated periodic syndrome (CAPS). Ann Rheum Dis 2016; Published Online First 4 October 2016. doi:10.1136/annrheumdis-2016-209686

2 Hoffman HM, Wanderer AA. Inflammasome and IL-1beta-mediated disorders. Curr Allergy Asthma Rep 2010;10:229-35.

3 Aróstegui Jl, Lopez Saldaña MD, Pascal M, et al. A somatic NLRP3 mutation as a cause of a sporadic case of chronic infantile neurologic, cutaneous, articular syndrome/neonatal-onset multisystem inflammatory disease: novel evidence of the role of low-level mosaicism as the pathophysiologic mechanism underlying Mendelian inherited diseases. Arthritis Rheum 2010;62:1158-66. 